\title{
Application of reverse-phase HPLC to quantify oligopeptide acetylation eliminates interference from unspecific acetyl CoA hydrolysis
}

\author{
Rune Evjenth${ }^{1}$, Kristine Hole ${ }^{1,2,3}$, Mathias Ziegler ${ }^{1}$ and Johan R Lillehaug*1
}

Address: ${ }^{1}$ Department of Molecular Biology, University of Bergen, N-5020 Bergen, Norway, ${ }^{2}$ Department of Surgical Sciences, University of Bergen, N-5020 Bergen, Norway and ${ }^{3}$ Department of Surgery, Haukeland University Hospital, N-5021 Bergen, Norway

Email: Rune Evjenth - rune.evjenth@mbi.uib.no; Kristine Hole - kristine.hole@mbi.uib.no; Mathias Ziegler - mathias.ziegler@mbi.uib.no; Johan R Lillehaug* - Johan.lillehaug@mbi.uib.no

* Corresponding author

from NAT 2007 and 2008 Symposia: Protein N-terminal Acetylation and Protein N-terminal Acetyltransferases (NATs)

Bergen, Norway. 24-25 May 2007 and II-13 September 2008

Published: 4 August 2009

BMC Proceedings 2009, 3(Suppl 6):S5 doi:10.1186/1753-656I-3-S6-S5

This article is available from: http://www.biomedcentral.com/I753-656I/3/S6/S5

(C) 2009 Evjenth et al; licensee BioMed Central Ltd.

This is an open access article distributed under the terms of the Creative Commons Attribution License (http://creativecommons.org/licenses/by/2.0),

which permits unrestricted use, distribution, and reproduction in any medium, provided the original work is properly cited.

\begin{abstract}
Protein acetylation is a common modification that plays a central role in several cellular processes. The most widely used methods to study these modifications are either based on the detection of radioactively acetylated oligopetide products or an enzyme-coupled reaction measuring conversion of the acetyl donor acetyl CoA to the product CoASH. Due to several disadvantages of these methods, we designed a new method to study oligopeptide acetylation. Based on reverse phase HPLC we detect both reaction products in a highly robust and reproducible way. The method reported here is also fully compatible with subsequent product analysis, e.g. by mass spectroscopy. The catalytic subunit, $\mathrm{hNaa30p}$, of the human $\mathrm{NatC}$ protein $\mathrm{N}$-acetyltransferase complex was used for $\mathrm{N}$-terminal oligopeptide acetylation. We show that unacetylated and acetylated oligopeptides can be efficiently separated and quantified by the HPLC-based analysis. The method is highly reproducible and enables reliable quantification of both substrates and products. It is therefore well-suited to determine kinetic parameters of acetyltransferases.
\end{abstract}

\section{Background}

Acetylation of proteins is a common protein modification that occurs either in the $\mathrm{N}$-terminal $\alpha$ amino group $\left(\mathrm{N}_{-}\right.$ acetylation) or the $\varepsilon$ amino group of lysine residues $\left(\mathrm{N}_{-}\right.$ acetylation). The corresponding acetylation reactions are catalysed by $\mathrm{N}^{\alpha}$-acetyltransferases (NATs) or histone acetyltransferases/lysine acetyltransferases (HATs/KATs), respectively $[1,2]$.

The important biological functions of protein acetylation have promoted extensive functional studies of different acetyltransferases to determine their kinetic properties, substrate specificities and catalytic mechanisms. For most of the enzymatic analyses, two different kinds of acetyl transfer assays are used. One uses radioactively labelled acetyl CoA as substrate [3]. The generation of radioactively labelled oligopeptides is monitored by a filter-binding assay and liquid scintillation counting [3]. This assay is very sensitive [4], but due to the use of radioactivity, the assay represents potential environmental and health risks and it is therefore relatively demanding to perform due to safety precautions. The other commonly used method is a 
spectrophotometric assay that continuously measures the amount of CoASH generated by the acetyltransferase reactions [5]. The CoASH is determined by a coupled enzyme system using either $\alpha$-ketoglutarate dehydrogenase or pyruvate dehydrogenase. The CoASH dependent oxidation of $\alpha$-ketoglutarate or pyruvate is coupled to the reduction of $\mathrm{NAD}^{+}$to $\mathrm{NADH}$, which is determined spectrophotometrically at $340 \mathrm{~nm}$. This assay is relatively inexpensive and can be performed with standard spectrophotometric equipment. A disadvantage of both methods is the difficulty to detect whether an oligopeptide substrate contains more than one lysine target residue.

Using the production of CoA as the basis for measuring acetyltransferase activity is linked to another potentially severe cause of error. In nearly all KAT assays, measuring the $\mathrm{N}^{\varepsilon}$-acetylation of lysines, a substantial amount of acetyl CoA will spontaneously react with the $\varepsilon$ amino group on lysine side chains, making it necessary to design proper controls to correct for this effect [4].

In addition, using the CoA-NADH coupled enzyme assay, it is not possible to use CoA as inhibitor to study acetyltransferase catalytic mechanism and, similarly to the filter assay, when more than one lysine target is present in the oligopeptide substrate, detailed acetylation site specificity can not be studied. To determine $\mathrm{Km}$ and Vmax values, the initial reaction rate must be determined under conditions giving linear initial reaction rates over the range of substrate concentration used, normally the substrate conversion should not exceed 10 - 15\% [4]. Furthermore, build-up of high concentrations of products may cause product inhibition. It is therefore of interest to obtain detailed information both on substrate consumption and product production. The radioactivity-based filter assay and the coupled-enzyme assay do not provide information on product consumption and detailed control experiments must be added. In the present article, we present a simple method for studying oligopeptide acetylation, using reverse phase HPLC detecting acetylated oligopeptides, in addition to CoASH. This method uses semi-automated HPLC-technology providing a fast, sensitive and highly reproducible assay for protein acetyltransferases. The instrument records the UV spectrum between 200 and $300 \mathrm{~nm}$, thereby enabling to monitor acetyl CoA and CoA $(260 \mathrm{~nm})$ and the peptide substrate and acetylated oligopeptides $(215 \mathrm{~nm})$ simultaneously. In addition, a radioactive flow detector may be connected to detect ${ }^{14} \mathrm{C} /{ }^{3} \mathrm{H}$ acetyl-oligopeptides to increase sensitivity, if required.

After the peptide separation using reverse phase HPLC, the relevant absorbance peaks are integrated and the corresponding areas are converted to amounts of product formed. The continuous recording of the UV spectra allows for each peak to be evaluated for the maximum absorbance wavelength which can be used to determine the purity of each absorbance signal.

Since the oligopeptide separation is performed in buffers containing acetonitrile and TFA, the HPLC method is also fully compatible with subsequent analytical procedures such as mass spectrometry to determine the site of modification.

\section{Methods \\ Chemicals}

Chemicals used in this study that are important for correct enzymatic determination are acetonitrile - ACN (Merck), trifluoracetic acid - TFA (Roche), acetyl CoA (Sigma), [1${ }^{14} \mathrm{C}$ ] acetyl CoA (56 mCi/mmol - GE Healthcare). All reagents were of analytical grade. Custom made oligopeptides (Table 1) were purchased from BioGenes, Germany.

\section{Cloning, expression and purification of MBP-hNaa30p}

The catalytic subunit of the human NatC complex; hNaa30p (earlier known as hMak3), a NAT acetyltransferase, was used to establish the reverse phase HPLC method. hNAA30 was cloned into the prokaryotic expression system pETM-MBP (originally obtained from G. Stier - EMBL, Heidelberg, Germany) and expressed in E. coli. The cloning, expression and purification were performed as described [6].

Table I: Oligopeptides used as substrates in this study

\begin{tabular}{|c|c|c|}
\hline Oligopeptide sequence & Abbreviation & Protein name $^{a}$ \\
\hline [H]MLGTEGG RWGRPVGRRRRPVRVYP [OH]* & ${ }_{1}$ MLGTE-RRR $_{24}$ & hnRNP H; (P31943) \\
\hline$[\mathrm{H}]$ MLALISR RWGRPVGRRRRPVRVYP [OH]* & ,MLAL-RRR 24 & hARL8b; (Q9NVJ2) \\
\hline$[\mathrm{H}]$ MLGTGPA RWGRPVGRRRRPVRVYP $[\mathrm{OH}]^{*}$ & ,MLGTG-RRR & mTOR; (P42345) \\
\hline$[\mathrm{H}]$ MLGPEGG RWGRPVGRRRRPVRVYP [OH]* & ,MLGP-RRR ${ }_{24}$ & hnRNP F; (P52597) \\
\hline
\end{tabular}

* Bold indicates amino acids found in corresponding protein while abbreviation indicate the names used in the text. The amino acid residues 8 to 24

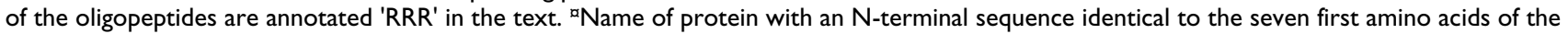
oligopeptide. 


\section{Separation of non acetylated and acetylated oligopeptides} using reverse phase HPLC

The acetylation activity was analysed by a reverse phase HPLC system, consisting of a LC-20AB solvent delivery module, an SPD-M20A photodiode array detector and a SIL-20AC autosampler (Shimadzu Prominence), and a $250 \mathrm{~mm} \times 3 \mathrm{~mm}$ Nucleosil C18 HD column (MachereyNagel) reverse phase HPLC column. In addition, a radioactivity flow detector (LB 509 - Berthold) and a peristaltic pump were connected down stream of the HPLC absorbance detector (Figure 1). All absorbance signals were quantified by integrating the peak of interest using the software LCSolution Version 1.21 SP1.

Prior to sample injection, the column was equilibrated for 5 minutes $(0.35 \mathrm{ml} / \mathrm{min}$ flow rate) with buffer A ( $5 \%$ acetonitrile (ACN) and $0.1 \%$ trifluoracetic acid (TFA)). After sample injection, the column was washed for 8 minutes with $2 \%$ elutionbuffer B ( $90 \%$ ACN, $0.1 \%$ TFA). The oligopeptides were then eluted employing a 40 minutes linear gradient from $2 \%$ to $40 \%$ buffer B. The column was then rinsed with $95 \%$ buffer B for 5 minutes. Finally, a 2 minutes linear gradient to $2 \%$ buffer $B$ was performed.

\section{Determination of steady-state kinetic constants with reverse phase HPLC}

$80 \mathrm{nM}$ of purified MBP-hNaa30p with $200 \mu \mathrm{M}$ 1 MLGTE$\mathrm{RRR}_{24}$ oligopeptide and $300 \mu \mathrm{M}$ acetyl CoA in acetylation buffer (50 mM Tris-HCl (pH 8.5), 10\% Glycerol, $1 \mathrm{mM}$ EDTA) were incubated for 60 minutes at $37^{\circ} \mathrm{C}$. Samples were collected after $0,10,20,30$, and 60 minutes incubation and analyzed by reverse phase HPLC.

To determine the $\mathrm{K}_{\mathrm{m} \text { oligopeptides }} 80 \mathrm{nM}$ of purified MBP$\mathrm{hNaa30p}$ was incubated with varying concentrations of oligopeptides ( 30 to $350 \mu \mathrm{M}$ ) and $300 \mu \mathrm{M}$ acetyl CoA in acetylation buffer for 30 minutes at $37^{\circ} \mathrm{C}$. When determining the $\mathrm{K}_{\mathrm{m} \text { acetyl CoA }} 300 \mu \mathrm{M}$ of ${ }_{1} \mathrm{MLAL} \mathrm{RRR}_{24}$ peptide was used in combination with varying 'concentrations of

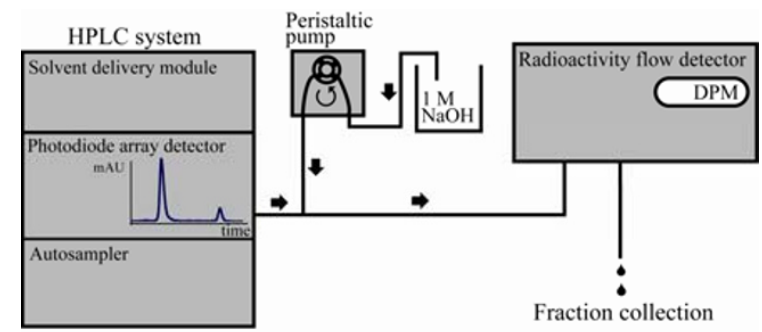

Figure I

Schematic presentation of the HPLC system included the equipment needed to detect the radioactivity. acetyl CoA ( 4 to $40 \mu \mathrm{M}$ ). The enzyme reactions were stopped by adding TFA to final concentrations of $1 \%(\mathrm{v} /$ $v)$. The amounts of acetylated oligopeptides were determined based on the absorbance at $215 \mathrm{~nm}$, while the production of CoA was determined by using the absorbance at $260 \mathrm{~nm}$. The steady-state enzyme kinetic parameters were calculated by nonlinear regression analysis using the SigmaPlot Technical Graphing Software (SPSS Inc.) The normality tests for all $\mathrm{Km}$ determinations were passed with value $>0.8$.

To verify the elution time for the acetylated oligopeptides, we conducted a time dependent acetylation assay by incubating purified MBP-hNaa30p (80 nM) with the oligopeptide ${ }_{1} \mathrm{MLGTE}^{-\mathrm{RRR}_{24}}(200 \mu \mathrm{M})$ and $\left[1-{ }^{14} \mathrm{C}\right]$ acetyl CoA (final concentration $300 \mu \mathrm{M}$ with specific activity $11.2 \mathrm{mCi} / \mathrm{mmol}$ ). Samples were collected after 0, 10, 20, 30 , and 60 minutes, placed on ice and adjusted to $1 \%$ TFA.

\section{Results}

\section{The HPLC system}

To establish the chromatographic procedure, first, the elution times for unmodified oligopeptides were determined by injecting $3 \mathrm{nmol}$ of pure oligopeptides diluted in the acetylation buffer on the HPLC system. Then we tested whether unacetylated and acetylated oligopeptides could be efficiently separated. We incubated an oligopeptide that is expected be a good hNaa30p substrate [7]; ${ }_{1}$ MLGTE-RRR $_{24}(200 \mu \mathrm{M})$ and $300 \mu \mathrm{M}$ acetyl CoA with 80 $\mathrm{nM}$ of purified MBP-hNaa30p in the acetylation buffer. The sample was incubated at $37^{\circ} \mathrm{C}$ with aliquots collected after $0,10,20,30$, and 60 minutes (see Methods). From the elution profile at Abs $215 \mathrm{~nm}$, we observed, as expected, a large amount of unmodified oligopeptide (Figure 2A, peak annotated 'a') and an additional peak with delayed elution time which increased with enzyme reaction time (Figure $2 \mathrm{~A}$ and $2 \mathrm{~B}$, peak annotated ' $\mathrm{b}$ '). The changes in absorbance profiles recorded at $260 \mathrm{~nm}$, detecting CoA and acetyl CoA in the same run, showed the same tendencies as the Abs $215 \mathrm{~nm}$ signals (Figure 3A and 3B). We observed a large excess of acetyl CoA (Figure 3A, peak annotated ' $b$ ') and an additional peak that increases during the course of the reaction (Figure $3 \mathrm{~A}$ and $3 \mathrm{~B}$, peak annotated ' $a$ '). The elution times of acetyl CoA and CoA were determined by injecting $3 \mathrm{nmol}$ of CoA or acetyl CoA and recording the resulting absorbance profiles. This analysis showed that CoA and acetyl CoA eluted after 6 minutes and 9 minutes and 30 seconds, respectively, peaks 'a' and ' $\mathrm{b}$ ' in Figure 3A.

\section{Analysing the sensitivity of the method}

The sensitivity of the HPLC based analysis was studied by injecting different amounts of oligopeptide and recording the resulting Abs $215 \mathrm{~nm}$ signal. The sensitivity for acetyl 
A

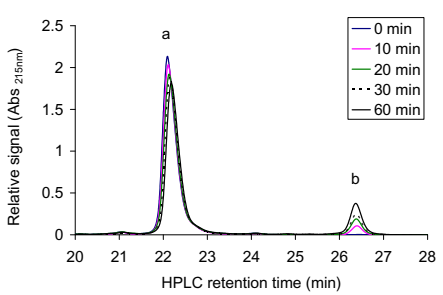

B

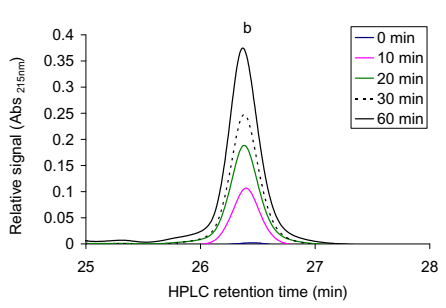

Figure 2

Reverse phase HPLC absorbance profile at 2 I $5 \mathrm{~nm}$ for the separation of acetylated and non-acetylated peptides. A; The oligopeptide ,MLGTE-RRR $24(200 \mu \mathrm{M})$ was incubated with acetyl CoA $(300 \mu \mathrm{M})$ and purified MBPhNa30p $(80 \mathrm{nM})$ in acetylation buffer for 60 minutes at $37^{\circ} \mathrm{C}$. Samples were collected at indicated time points and analysed with reverse phase HPLC. The resulting absorbance profile at $215 \mathrm{~nm}$ indicate good separation of unacetylated ('a') and acetylated oligopeptides ('b'). B; An expanded version of the absorbance profile for the formation of acetylated oligopeptide. A clear time dependent increase in the absorption signal is observed.

CoA was determined by injecting different amounts of acetyl CoA and recording the resulting Abs $260 \mathrm{~nm}$ signal. A linear correlation between the absorbance signals and the amount of substrates added was observed (Figure 4A and $4 \mathrm{~B}$ ). We noted that $0.5 \mathrm{nmol}$ was the lower limit for reliable quantification of oligopeptides at $215 \mathrm{~nm}$, which corresponded to $5 \mu \mathrm{M}$ in a reaction volume of $100 \mu \mathrm{l}$.

To enhance the detection sensitivity and to verify that the novel delayed absorption peak represented oligopeptides with one added acetyl group, we connected a radioactive flow detector after the absorbance detector (Figure 1). This allowed us to use radioactively labelled acetyl CoA as acetyl donor. To prevent background accumulation of radioactivity in the solid scintillator detector system, $1 \mathrm{M}$ $\mathrm{NaOH}$ was mixed to the column effluent in a 1:10 mixing ratio by connecting a peristaltic pump in-line between the absorbance detector and the radioactivity flow detector (Figure 1). High concentration of $\mathrm{NaOH}$ was used so that the dilution of the samples, thus dilution of the radioactive signals was as low as possible. Using [1-14 C] acetyl
A

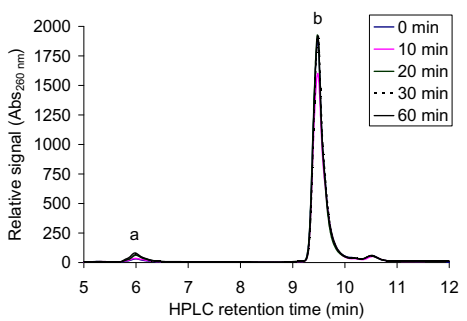

B

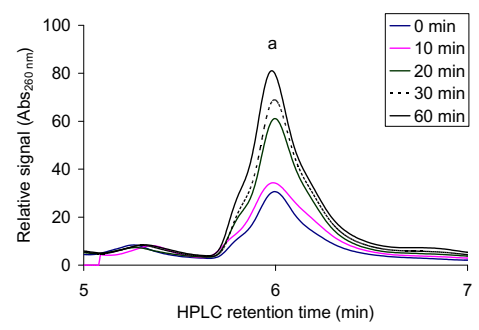

\section{Figure 3}

Reverse phase HPLC absorbance profile of the separation of CoA and acetyl CoA. A; The oligopeptide ${ }_{\text {IMLGTE-RRR }}$ ML $_{2}(200 \mu \mathrm{M})$ was incubated with acetyl CoA $(300 \mu \mathrm{M})$ and purified MBP-hNaa30p $(80 \mathrm{nM})$ in acetylation buffer for 60 minutes at $37^{\circ} \mathrm{C}$. Samples were collected at indicated time points and analysed with reverse phase HPLC. The resulting absorbance profile at $260 \mathrm{~nm}$ indicates good separation of CoA ('a') and acetyl CoA ('b'). B; An expanded version of the absorbance profile for the formation of CoA. A time dependent increase in the absorption signal is observed.

CoA as acetyl donor in the reaction, we observed a similar increase in radioactively labelled acetylated oligopeptides (Figure 5) as observed for the Abs $215 \mathrm{~nm}$ absorption signal (Figure 2B, peak ' $b$ '). This verifies that the peptide with delayed elution time is labelled with radioactive acetyl groups and that the quantity of the signal increased with enzyme reaction time.

\section{Quantification of CoA has the potential of generating false kinetic data}

Our results indicated that the HPLC system is a solid method to study peptide acetylation. Using the non-radioactive HPLC method to detect the amount of acetylated oligopeptides, we determined hNaa30p enzyme kinetic constants for some in vitro oligopeptide substrates. Different concentrations of ${ }_{1}$ MLGTG-RRR 24 peptides (30-350 $\mu \mathrm{M})$ were used with fixed concentration of acetyl CoA $(300 \mu \mathrm{M})$ and $80 \mathrm{nM}$ of purified MBP-hNaa30p. When using the absorption signal at $215 \mathrm{~nm}$, representing acetylated oligopeptides, we calculated the $\mathrm{K}_{\mathrm{m}}$ oligopeptide to be $283 \mu \mathrm{M}$ with Vmax of $3.3 \mathrm{pmol}^{*} \mathrm{~min}^{-1}{ }^{*} \mathrm{pmol}$ hNaa30 $\mathrm{p}^{-1}$ (Table 2). In the same run, the production of CoA was recorded at $260 \mathrm{~nm}$ and used to calculate the cor- 


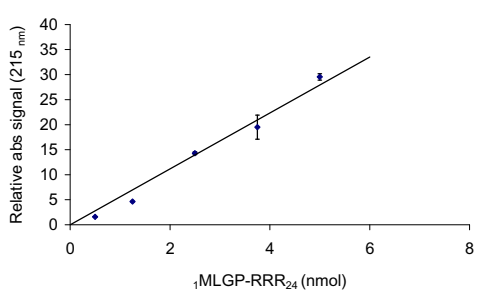

B

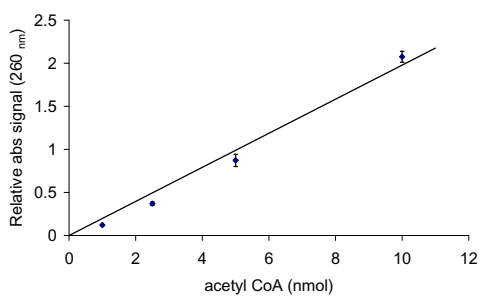

Figure 4

Standard curves of increasing amount of the substrate ,MLGP-RRR 24 and acetyl CoA analysed by reverse phase HPLC. A; Five different amounts of the oligopeptide IMLGP-RRR24, diluted in acetylation buffer, were analysed by reverse phase HPLC. The resulting absorption signals at $215 \mathrm{~nm}$ were quantified. Each amount were analysed three times and error bars indicate S.D. B; Five different amounts of acetyl CoA, diluted in acetylation buffer, were analysed by reverse phase HPLC. The resulting absorption signals at $260 \mathrm{~nm}$ were quantified. Each amount were analysed three times and error bars indicate S.D.

responding kinetic constants. Here we observed that the Michaelis Menten plot based on CoA production generated a dose dependent curve from which a significantly lower Vmax was obtained (Figure 6) compared to when Vmax was calculated based on the production of acetylated oligopeptides (Figure 7 ). $\mathrm{K}_{\mathrm{m} \text { oligopeptide }}$ based on CoA production was determined to be $3.1 \mu \mathrm{M}$ with a Vmax of $8.7 \mathrm{pmol}^{*} \min ^{-1}{ }^{*}$ pmol hNaa30p ${ }^{-1}$ (Table 2). It is important to note that approximately eight pmoles CoASH (Figure 6) were produced per pmole acetylated oligopeptide (Figure 7). Since theoretically one mole CoASH should be generated per mole acetylated oligopeptide, a discrepancy in CoASH production relative to acetylated oligopeptide was apparent. Km for acetyl CoA

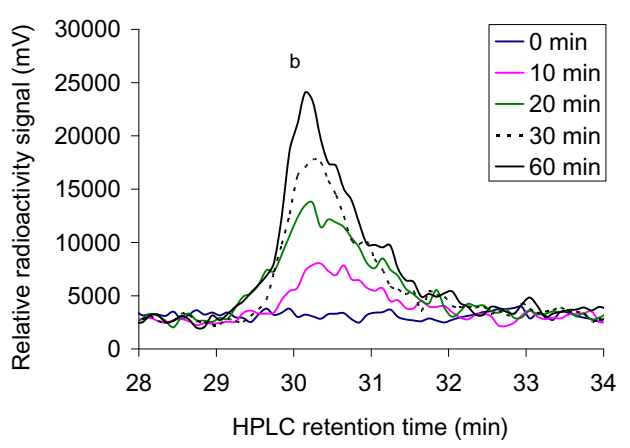

\section{Figure 5}

The radioactive signal from oligopeptides being modified with radioactive acetyl CoA. The oligopeptide ${ }_{1}$ MLGTE-RRR $_{24}(200 \mu \mathrm{M})$ was incubated with [I-14C] acetyl CoA (final $300 \mu \mathrm{M}$ with specific activity $11.2 \mathrm{mCi} / \mathrm{mmol}$ ) and purified MBP-hNaa30p $(80 \mathrm{nM})$ in acetylation buffer for 60 minutes at $37^{\circ} \mathrm{C}$. Samples were collected at indicated time points and analysed with reverse phase HPLC that had been connected to a radioactivity flow detector after the absorbance detector. A clear time dependent increase in the radioactivity signal is observed, verifying that the eluted oligopeptides are labelled with radioactive acetyl groups.

were with non linear regression determined to be approximately $14 \mu \mathrm{M}$ with a Vmax of $2.1 \mathrm{pmol}^{*} \mathrm{~min}^{-1}{ }^{*} \mathrm{pmol}$ hNaa30p-1 (Figure 8). V/K for selected substrates was calculated (Figure 9) and the S.D., indicated by error bars, were determined based on three independent experiments. The data demonstrate that substrate selectivity of the enzyme can be readily detected by the HPLC method.

\section{Discussion and conclusion}

The radioactivity-based filter assay [3] and the CoANADH coupled enzyme assay [5] are the most commonly applied methods to study acetyltransferase kinetics and mechanisms. Both these methods suffer from significant drawbacks such as biohazard and non-enzymatic deacetylation of acetyl CoA. To eliminate these problems and to allow us to analyse the acetylated oligopeptide products by mass spectrometry, we developed a method for studying peptide acetylation based on reverse phase

Table 2: Comparison of $K_{m}$ oligopeptide and $V m a x$ based on the detection of $\operatorname{CoA}_{(260 \mathrm{~nm})}$ and the detection of acetylated oligopeptides $(215$ nm)

\begin{tabular}{|c|c|c|c|}
\hline \multicolumn{2}{|r|}{ CoA } & \multicolumn{2}{|r|}{ Ac-, MLGTG-RRR ${ }_{24}$} \\
\hline $\mathrm{Km}(\mu \mathrm{M})$ & Vmax $\left(\right.$ pmol product $* \min ^{-1} *$ pmol hNaa30 $\left.\mathrm{p}^{-1}\right)$ & $\mathrm{Km}(\mu \mathrm{M})$ & Vmax $\left(\right.$ pmol product $* \min ^{-1} *$ pmol hNaa30 $\left.\mathrm{p}^{-1}\right)$ \\
\hline 3.1 & 8.7 & 283.2 & 3.3 \\
\hline
\end{tabular}


A

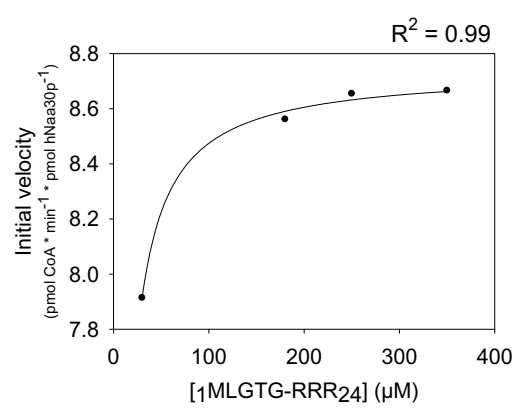

B

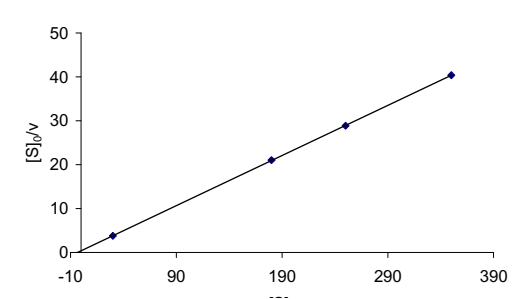

Figure 6

Determination of $\mathrm{Km}$ and $\mathrm{Vmax}$ for the , MLGTG$\mathbf{R R R}_{24}$ oligopeptide, based on the generation of CoA. Purified MBP-hNaa30p (80 nM) was incubated with $300 \mu \mathrm{M}$ acetyl CoA and varying concentrations of ${ }_{1} M L G T G-R R R_{24}$ $(30-350 \mu \mathrm{M})$ in acetylation buffer for 30 minutes at $37^{\circ} \mathrm{C}$. A; Non linear regression analysis of the dose dependent curve generated based on the analysis of the CoA absorption signal at $260 \mathrm{~nm}$. The coefficient of determination $\left(R^{2}\right)$ is given above the plot. B; Hanes-Woolf plot of the dose dependent acetylation signal.

HPLC. After acetylation, non-acetylated and acetylated oligopeptides are separated and quantified by integrating the respective elution peaks.

Since the first residues of the substrates seem to be most important for enzyme specificity [1], we designed peptides that deviated only within the 7 first $\mathrm{N}$-terminal positions. The next 17 amino acids that are indicated by 'RRR' are identical in all peptides and resemble the sequence of Adrenocorticotropic hormone (ACTH) (Amino acid no. 8 to 24), but all Lys residues were replaced with Arg to minimize aberrant $\mathrm{N}-\varepsilon$ acetylation. The separation of peptides were carried out with $0.1 \%$ TFA in the HPLC buffers, making the peptide residues highly protonated. The positively charged Arg residues facilitate peptide solubility and separation by reverse phase HPLC. The N-terminal acetylation substitutes a positive charge by a hydrophobic group, causing the acetylated oligopeptides to be separated from the non acetylated form due to stronger interaction with the Nucleosil C18 HD matrix, resulting in increased elution time. Several acetylation assays with other $\mathrm{N}^{\alpha}$-acetyltransferases acetylating oligopeptides containing more
A

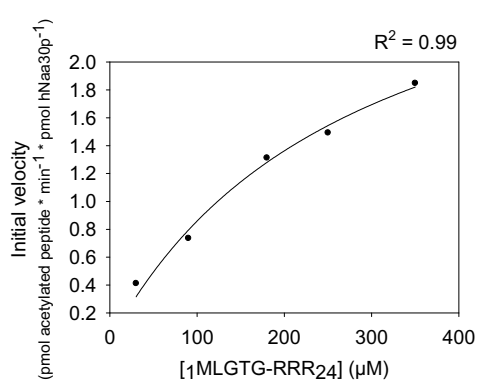

B

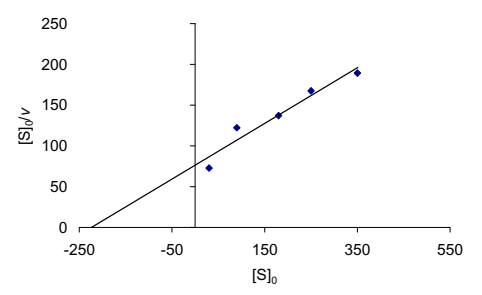

Figure 7

Determination of $\mathrm{Km}$ and Vmax for the , MLGTG$\mathbf{R} \mathbf{R}_{\mathbf{2 4}}$ oligopeptide, based on the generation of acetylated , MLGTG-RRR 24 oligopeptide. Purified MBPhNa330p ( 80 nM) was incubated with $300 \mu \mathrm{M}$ acetyl CoA and varying concentrations of MLGTG-RRR $_{24}(30-350 \mu \mathrm{M})$ in acetylation buffer for 30 minutes at $37^{\circ} \mathrm{C}$. A; Non linear regression analysis of the dose dependent curve generated based on the analysis of the acetylated oligopeptide absorption signal at $215 \mathrm{~nm}$. The coefficient of determination $\left(R^{2}\right)$ is given above the plot. B; Hanes-Woolf plot of the dose dependent acetylation signal.

hydrophobic residues, showed that the acetylated form of these oligopeptides also could be efficiently separated with the reverse phase Nucleosile C18HD column. This indicates that commercially available oligopeptide substrates, composed of the endogenous amino acids, can be used as substrates with this detection method.

The unacetylated and acetylated oligopeptide ${ }_{1}$ MLGTE$\mathrm{RRR}_{24}$ was separated by more than 3 minutes. Even when using high amounts of oligopeptides, >30 nmols, leading to a widening of the peaks, an efficient separation of unacetylated and acetylated oligopeptides was achieved. If necessary, the separation of oligopeptides can be further enhanced by optimising the elution profile.

The sensitivity of the detection method was determined by injecting different amount of oligopeptides and acetyl CoA and quantifying the resulting absorption profiles. This showed a clear linear trend for both substrates, spanning from 0.5 to $5 \mathrm{nmol}$ of acetyl CoA and from 1 to 10 nmol of oligopeptide. The coefficient of determination 
A

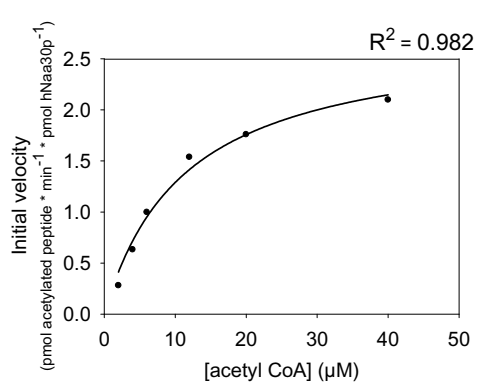

B

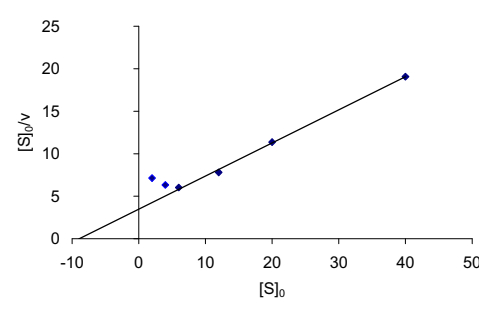

\section{Figure 8}

Determination of $\mathrm{Km}$ and Vmax for acetyl CoA, based on the generation of acetylated , MLAL-RRR 24 oligopeptide. Purified MBP-hNaa30p (80 nM) was incubated with ,MLAL-RRR 24 oligopeptide at saturated levels and varying concentrations of acetyl CoA $(4-40 \mu \mathrm{M})$ in acetylation buffer for 30 minutes at $37^{\circ} \mathrm{C}$. A; Non linear regression analysis of the dose dependent curve generated based on the analysis of the acetylated oligopeptide absorption signal at $215 \mathrm{~nm}$. The coefficient of determination $\left(R^{2}\right)$ is given above the plot. B; Hanes-Woolf plot of the dose dependent acetylation signal.

$\left(R^{2}\right)$ was above 0.97 for both substrates. Our experience with this acetylation assay is that even at very high amounts of oligopeptides, up to $30 \mathrm{nmol}$, the increase in absorbance at $215 \mathrm{~nm}$ is linear with coefficient of determination above 0.97 (data not shown).

The reproducibility of the method was analysed by calculating the standard deviation (S.D.) from three independent experiments. This was done for the sensitivity determination and the calculation of the kinetic constants. The result demonstrated that the reverse phase HPLC method is highly reproducible when analysing acetylation based on the detection of acetylated oligopeptides with the Abs $215 \mathrm{~nm}$ signal.

In conclusion, we have established a robust and highly reproducible method for studying oligopeptide acetylation. With new semi-automated reverse phase HPLC technology, we show that both substrates, acetyl CoA and unacetylated oligopeptides, and enzyme products, CoA and acetylated oligopeptides can be detected and quanti-

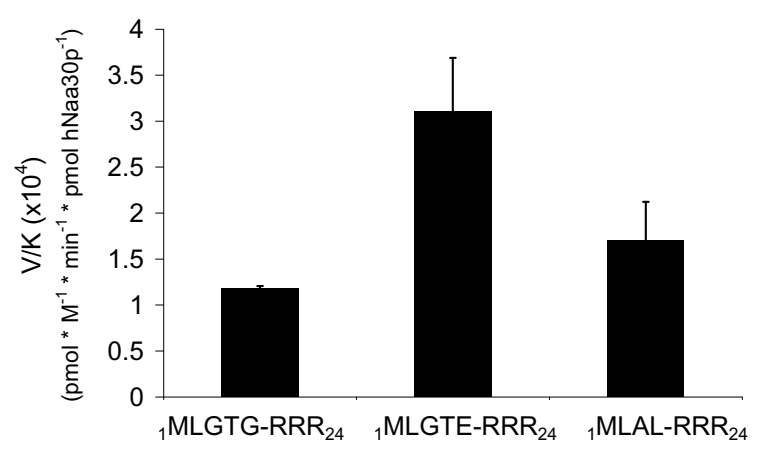

\begin{abstract}
Figure 9
MBP-hNaa30p specificity constants (V/K) for selected oligopeptides based on detection of acetylated oligopeptides $_{(215 \mathrm{~nm})}$. Purified MBP-hNaa30p (80 nM) was incubated with selected oligopeptides and acetyl CoA (300 $\mu \mathrm{M})$ in acetylation buffer for 30 minutes at $37^{\circ} \mathrm{C}$. The acetylation kinetics was analysed by reverse phase HPLC. V/ $\mathrm{K}$ is the $\mathrm{V}_{\text {max }} / \mathrm{K}_{\mathrm{m} \text { (oligopeptides). }}$. Error bars indicate S.D. Experiments are performed in triplicates.
\end{abstract}

fied in the same experiment. This allows for increased control over substrate conversion and product generation forming a solid basis for data intepretation. Importantly, the assay is easy to perform and automation reduces sample handling.

\section{Competing interests}

The authors declare that they have no competing interests.

\section{Authors' contributions}

RHE and JRL designed the study. MZ and RHE proposed and optimized the method. RHE and KH conducted the experimental work. RHE and JRL participated in the data analysis and writing of the manuscript. All authors have read and approved the final manuscript.

\section{Acknowledgements}

We thank L. Vikebø, M. Algroy and N. Glomnes for technical assistance.

This article has been published as part of BMC Proceedings Volume 3 Supplement 6, 2009: Proceedings of the 2007 and 2008 Symposia on Protein $\mathrm{N}$-terminal Acetylation. The full contents of the supplement are available online at http://www.biomedcentral.com//753-656I/3? issue=S6

\section{References}

I. Polevoda B, Sherman F: N-terminal acetyltransferases and sequence requirements for $\mathbf{N}$-terminal acetylation of eukaryotic proteins. J Mol Biol 2003, 325(4):595-622.

2. Marmorstein R: Biochemical and structural characterization of recombinant histone acetyltransferase proteins. Methods Enzymol 2004, 376:106-119.

3. Tanner KG, Trievel RC, Kuo MH, Howard RM, Berger SL, Allis CD, Marmorstein R, Denu JM: Catalytic mechanism and function of invariant glutamic acid 173 from the histone acetyltrans- 
ferase GCN5 transcriptional coactivator. J Biol Chem 1999, 274(26): $18157-18160$.

4. Berndsen CE, Denu JM: Assays for mechanistic investigations of protein/histone acetyltransferases. Methods 2005, 36(4):32I-33I.

5. Kim Y, Tanner KG, Denu JM: A continuous, nonradioactive assay for histone acetyltransferases. Anal Biochem 2000, 280(2):308-3।4.

6. Arnesen T, Kong X, Evjenth R, Gromyko D, Varhaug JE, Lin Z, Sang $\mathrm{N}$, Caro J, Lillehaug JR: Interaction between HIF-I alpha (ODD) and hARDI does not induce acetylation and destabilization of HIF-I alpha. FEBS Lett 2005, 579(28):6428-6432.

7. Starheim KK, Gromyko D, Evjenth R, Ryningen A, Varhaug JE, Lillehaug JR, Arnesen T: Knockdown of the Human N\{alpha\}-Terminal Acetyltransferase Complex C (hNatC) Leads to p53Dependent Apoptosis and Aberrant hArl8b Localization. Mol Cell Biol 2009, 29:3569-358I.

Publish with Bio Med Central and every scientist can read your work free of charge

"BioMed Central will be the most significant development for disseminating the results of biomedical research in our lifetime. "

Sir Paul Nurse, Cancer Research UK

Your research papers will be:

- available free of charge to the entire biomedical community

- peer reviewed and published immediately upon acceptance

- cited in PubMed and archived on PubMed Central

- yours - you keep the copyright

Submit your manuscript here:

http://www.biomedcentral.com/info/publishing_adv.asp 